\title{
Gestão do cuidado e matriciamento na atenção primária à saúde: um relato de experiência
}

\author{
Minéia da Costa Figueiredo, Fabiana Lopes de Paula
}

\section{RESUMO}

Este artigo irá abordar a vivência em uma unidade de Estratégia Saúde da Família (ESF) no município de Canto do Buriti-PI sobre os aspectos relacionados ao processo de matriciamento e a gestão do cuidado. A descrição será feita a partir da análise da gestão do cuidado e articulações com o Núcleo de Apoio à Saúde da Família (NASF) e suas ações de apoio matricial desenvolvidas na unidade. O NASF tem fundamental importância na resolução de casos mais complexos e na efetivação de cuidado integral, bem como para a assistência humanizada aos usuários na Atenção Primária à Saúde. $O$ relato traz como contribuição a análise do processo de trabalho entre eSF e NASF, com uma visão que traz subsídios para a reflexão; tendo a intenção de cooperar para o aperfeiçoamento, progresso das ações na APS e a concretização das políticas públicas.

Palavras-chave: Atenção Primária à Saúde; Estratégia Saúde da Família; Equipe de Assistência ao Paciente; Integralidade em Saúde; Sistema Único de Saúde.

\section{ABSTRACT}

This article will address the experience in a Family Health Strategy (ESF) unit in the city of Canto do Buriti-PI on aspects related to the matrix support process and care management. The description will be made from the analysis of care management and articulations with the Family Health Support Center (NASF) and its matrix support actions developed in the unit. The NASF is of fundamental importance in solving more complex cases and in providing comprehensive care, as well as in providing humanized assistance to users in Primary Health Care. The report contributes to the analysis of the work process between eSF and NASF, with a a vision that supports reflection; with the intention of cooperating for the improvement, progress of actions in the PHC and the implementation of public policies.

Keywords: Primary Health Care; Family Health Strategy; Patient Care Team; Integrality in Health; Unified Health System.
Revista da Rede APS 2021

Publicada em: 20/09/2021

DOI:10.14295/aps.v3i2.163

Minéia da Costa Figueiredo (Secretaria de Saúde, Canto do Buriti,-PI, Brasil)

Fabiana Lopes de Paula (Centro de Ciências da Saúde, Universidade Federal do Recôncavo da Bahia, Santo Antônio de Jesus, BA, Brasil)

Correspondência para:

Minéia da Costa Figueiredo mineiacostaf@gmail.com 


\section{INTRODUÇÃO}

A Atenção Básica é caracterizada por um leque de ações de saúde, na esfera individual e coletiva, que envolve a promoção e a proteção da saúde, a prevenção de agravos, o diagnóstico, o tratamento, a reabilitação, a redução de danos e a manutenção da saúde com a finalidade de desenvolver uma atenção integral que gere mudanças na situação de saúde e autonomia dos sujeitos e nos determinantes e condicionantes de saúde das coletividades (BRASIL, 2017).

Por estar no território do indivíduo, a Estratégia Saúde da Família (ESF) conhece seu contexto social, laboral, crenças, cultura, vulnerabilidades e potencialidades. Por isso, tem condições de desenvolver um cuidado integral, pautado nas necessidades reais dos indivíduos ou coletividades. Além disso, possui vínculos maiores com a população, sendo o contato preferencial dos sujeitos e coletividades. No entanto, algumas ações precisam ser referenciadas para outros pontos da Rede de Atenção à Saúde (RAS), para garantir a integralidade do cuidado, sendo indispensável a contra referência para que haja coordenação do cuidado e corresponsabilidade entre os diversos pontos da rede.

Basta ressaltar que para haver uma coordenação eficaz, é imprescindível que sejam consideradas as necessidades dos usuários, contexto dos sistemas de saúde, com suas diversas competências e formas de organização. Além disso, são necessários ajustes compatíveis com as demandas e singularidades de forma a oferecer uma resposta resolutiva aos problemas de saúde das populações (ALMEIDA, et al., 2018).

Uma das ferramentas para a garantia da integralidade e coordenação do cuidado é o apoio matricial (AM) nos limites da Atenção Primária à Saúde (APS). Este apoio é uma estratégia de coordenação do trabalho em saúde, a partir da necessidade de expandir os resultados da atuação da ESF, formada por uma equipe multiprofissional que tem como objetivo dar assistência e cuidados em saúde no território, de maneira interdisciplinar, potencializando a integralidade e a resolutividade dos atendimentos. Dessa forma, no processo de trabalho das ESF, o matriciamento auxilia na realização de ações possíveis e no diagnóstico, pontos importantes para a elaboração de um Projeto Terapêutico Singular (PTS) (SANTOS et al., 2017).

Assim, o AM é um arranjo organizacional e um método de compartilhamento de conhecimentos que permite o suporte técnico pedagógico, e institucional, garantindo retaguarda especializada para as equipes responsáveis pela construção de ações para a população adscrita, nomeadas Equipe de Referência (ER) (LAZARINO et al., 2019).

Diante do exposto, percebe-se o quanto é importante o apoio matricial e a gestão do cuidado na Atenção Primária à Saúde, para a resolutividade das ações e questões em tempo oportuno, como também diminuir encaminhamentos e procedimentos desnecessários. Ademais, precisa haver a garantia da continuidade da assistência à saúde, uma vez que o indivíduo sempre será do território.

Assim, diante da importância da APS em conjunto com o AM na garantia de cuidado de qualidade aos usuários do Sistema Único de Saúde, foi utilizada como questão norteadora deste estudo a seguinte pergunta: Como acontece a gestão do cuidado e articulações com o Núcleo de Apoio à Saúde da Família? Nesse sentido, o presente estudo tem como intuito analisar a gestão do cuidado na Atenção Básica em uma ESF do município de Canto do Buriti-PI.

\section{MÉTODOS}

Trata-se de um estudo descritivo, tipo relato de experiência que irá abordar a vivência em uma unidade de ESF sobre os aspectos relacionados ao processo de matriciamento e a gestão do cuidado, segundo a ótica da enfermeira responsável por essa ESF. A descrição será feita a partir da análise da gestão do cuidado e 
articulações com o Núcleo de Apoio à Saúde da Família (NASF) e suas ações de apoio matricial desenvolvidas na unidade no período de 2018 a 2019. Será abordado como acontece o fluxo de atendimentos, reuniões de equipe e discussões de caso, apoio matricial e outras ações que são desenvolvidas na rotina e que permitam analisar a gestão do cuidado.

$O$ relato descreve a experiência vivenciada em uma equipe de ESF do município de Canto do Buriti-PI, no Estado do Piauí com área territorial de $4.325,642 \mathrm{~km}^{2}$ e população estimada de 21.1897 (IBGE, 2019). Essa unidade de saúde possui uma população adscrita totalmente rural e abrange aproximadamente 1700 usuários, distribuídos em 15 áreas. A equipe de saúde de família é composta por 1 enfermeiro, 2 técnicas de enfermagem, 1 médico, 1 dentista, 1 técnica em saúde bucal, 1 digitadora e 8 agentes comunitários de saúde (ACS). Quanto ao NASF é composto por fisioterapeuta, assistente social, nutricionista, educador físico, farmacêutico e fonoaudiólogo.

\section{RESULTADOS E DISCUSSÃO}

\section{Importância do apoio matricial na integralidade do cuidado}

A ESF é formada por equipe multiprofissional e tem como objetivo ofertar atendimento de saúde que garanta cuidado integral, levando sempre em conta a complexidade deste indivíduo e coletividades no seu território adscrito. Contudo, estas concepções ainda não estão bem concretizadas na equipe de saúde da família, o que se tem observado muitas vezes é a predominância do modelo biomédico. Isso significa que há alta demanda de consultas e atendimentos voltados ao tratamento curativo, com um olhar pouco direcionado à promoção da saúde, embora esse seja o seu principal eixo. Há dificuldade de discussão de casos e produção de PTS na reunião de equipe, dificultando o planejamento de ações no cotidiano do serviço.
Isso acontece porque as demandas do cotidiano direcionadas às equipes de ESF no seu território provocam um leque de ações de cuidado. A alta complexidade do trabalho em APS é concebida pela construção de práticas de cuidado em saúde que vão desde atos protocolares de atividades programadas a ambulatoriais, passando pela assistência de famílias com riscos e vulnerabilidades, ações de promoção e educação em saúde, controle social, saúde mental e demandas dos programas de assistência social (MEDEIROS, 2015).

É nesse contexto que o NASF tem fundamental importância para o apoio técnico pedagógico, facilitando a resolução de casos mais complexos e colaborando na efetivação de cuidado integral, bem como para a assistência humanizada aos usuários na Atenção Primária à Saúde. Assim, ele oferece apoio especializado à ESF, trazendo o olhar de outras formações, aumentando a resolutividade de muitas situações. Entretanto, para que as práticas do NASF colaborem para o cuidado integral, precisam estar em consonância com a Política de Humanização que traz a clínica ampliada e compartilhada.

Lazarino e colaboradores (2019) relatam que na rede de serviços de APS do município de BetimMG, os profissionais das ESF consideram que o matriciamento proporcionou a ampliação do olhar sobre o trabalho em saúde. Neste estudo, o apoio matricial foi considerado determinante no processo saúde-doença, uma vez que aponta e reforça para a troca de experiências como sendo um caminho potente para a construção e reconstrução de conhecimento.

Corroborando, no estudo de Gonçalves e coparticipantes (2015) desenvolvido em São Paulo, as pactuações entre as equipes do NASF e eqSFs foram essenciais para que houvesse expansão do olhar técnico e do escopo de ações a serem realizadas, como também dividir a responsabilidade sobre algumas tarefas, por exemplo, grupos, oficinas, visitas domiciliares, entre outras. Esse modo de organização era próprio dos NASFs estudados, pois os profissionais acreditam que essa conformação fortalecia planejamentos e ações mais estruturadas. 
Assim, o apoio matricial se faz mais eficaz quando envolve o trabalho conjunto entre os profissionais da ESF e NASF, refletindo e operando nos casos, e extrapolam as atividades de educação permanente quando efetivadas isoladamente (FAGUNDES, et al., 2021).

Dessa forma, para que haja cuidado integral, as singularidades dos sujeitos e coletividades devem ser consideradas. Logo, é necessário o uso de ferramentas como a escuta qualificada, reunião de equipe, discussão de caso, elaboração do Projeto Terapêutico Singular (PTS) para os casos mais complexos, entre outras ações. Visto isso, a equipe matriciadora deve nortear suas ações com base na Clínica Ampliada, evitando exercer suas atividades de forma ambulatorial, com participação no processo de cuidado e garantia de apoio técnico-pedagógico às equipes (BRASIL, 2009).

No entanto, o núcleo de apoio que atua na ESF em questão tem suas ações deliberadas por uma coordenadora, que escala os profissionais para a unidade, como também as ações a serem desenvolvidas. Os profissionais fazem rodízios entre as unidades, o que dificulta a criação de vínculos e conhecimento da população adscrita. Desse modo, as ações desenvolvidas são pontuais e nem sempre parte das necessidades reais da população, o que contraria os conceitos da clínica ampliada que prevê a ampliação do "objeto de trabalho", que é o sujeito.

Percebe-se no processo de trabalho que a prática matricial não é desenvolvida, priorizando-se atendimentos individualizados e especializados. Portanto, é imprescindível que haja uma maior compreensão da gestão em relação ao trabalho desses profissionais, para que tenha priorização de ações desenvolvidas nas unidades de saúde que garantam um cuidado integral, permitam conhecer as vulnerabilidades e potencialidades, para se desenvolver um trabalho humanizado, centrado na pessoa e não na doença.

Uma das ferramentas que pode ser utilizada como espaço de discussão de casos e elaboração de PTS é a reunião de equipe. Silva e colaboradores (2017) descrevem que a compreensão das ferramentas de gestão da clínica, através de discussões nas reuniões de equipe e da prática da educação permanente, beneficia uma dinâmica de trabalho onde as visões de núcleo e campo ajudam a modificar e a transformar as ações de produção de cuidado para o modelo recomendado pelo SUS.

No entanto, como há dificuldade de unir a equipe multiprofissional e NASF esse momento não é utilizado para apoio matricial, e sim como espaço para discussão de processo de trabalho e entrega de produções. Isso impede a construção de ações importantes na resolução de casos, como respostas ágeis e assertivas às reais necessidades dos usuários. Logo, para reunir-se e construir um PTS, será indispensável instituir essa oportunidade nas atividades habituais da unidade, produzindo espaços na agenda, através da flexibilização nos horários dos profissionais da ESF e matriciadores.

Santos e colaboradores (2017) evidenciaram em sua pesquisa que existe diferença na operacionalização do trabalho de ESF e NASF, isso porque a organização do trabalho de médicos e enfermeiros é sustentada por agenda fixa de atendimentos, enquanto os outros profissionais se adaptam à equipe de saúde, sendo acionados de acordo com as necessidades do serviço. Este é um dos maiores desafios enfrentados pelas equipes NASF, que, além de construir um processo de trabalho até então inexistente na $A B$, precisa criar espaços e formas para desenvolver apoio e compartilhamento de ações (OLIVEIRA,; BADUY; MELCHIOR, 2019).

Essa dificuldade é encontrada neste estudo, além de outras, como espaço físico inadequado e falta de transporte para a realização de visitas domiciliares, fato que agrava os obstáculos encontrados, já que atualmente os serviços prestados por essa ESF não se desenvolvem em seu espaço físico adequado, que seria na zona rural, território dos indivíduos. O espaço físico atualmente está na zona urbana, assim as pessoas dependem de transportes para se deslocarem à Unidade Básica Saúde, muitas vezes não tendo condições suficientes para pagar esse deslocamento. Isso porque muitas possuem como renda apenas o bolsa família. 
Com isso, o acesso fica mais difícil à população por questões geográficas e financeiras, já que o deslocamento traz custos.

Diante dos fatos narrados, compreende-se que a atuação do AM e ER são determinadas pela organização e formato de gestão do trabalho em saúde. Estes necessitam ampliar as possibilidades da prática da clínica ampliada e da troca de saberes entre diferentes especialidades.

\section{Processo de trabalho NASF e ESF}

O cronograma do NASF é entregue mensalmente à enfermeira da ESF, sendo posteriormente apresentado em reunião de equipe e fixado no mural da unidade. Dessa forma, as demandas são encaminhadas ao NASF, através de guias de encaminhamento, como também fichas de referência e contrarreferência. A partir da análise da enfermeira foi constatado que os atendimentos realizados, não eram registrados em prontuários e nem discutidos com a equipe. Muitas vezes foi necessário o retorno do usuário ao serviço ou visita domiciliar para o conhecimento da consulta especializada, impedindo a readequação do PTS e consequentemente a integralidade do cuidado.

A mesma realidade foi encontrada em estudo realizado na Bahia, onde equipes de saúde da família utilizam instrumentos e vias identificadas para propiciar a comunicação, limitados à ficha de referência e contrarreferência ou contato telefônico. No entanto, esses instrumentos não lograram êxito no município, tendo em vista a técnica pouco frequente da contrarreferência. Também, evidenciou-se que as equipes confrontavam consideráveis dificuldades de comunicação, horizontal e vertical (ALELUIA et al., 2017)

Cabe ressaltar que a responsabilização compartilhada pelos casos visa potencializar a capacidade resolutiva da equipe local, estimulando a interdisciplinaridade e o alcance de novas competências. É importante também para que os usuários possam se responsabilizar pelo seu tratamento, sintomas e sua vida, produzindo outras relações com o seu processo de adoecimento (BRASIL, 2010).

Uma das limitações para o trabalho compartilhado vem da compreensão e vivência fragmentada do trabalho e do cuidado, referente ao modelo hegemônico de assistência com início na formação da maioria dos profissionais de saúde, não direcionada para o trabalho coletivo (OLIVEIRA; BADUY; MELCHIOR, 2019) .

É evidente a falha de comunicação entre as equipes, inviabilizando o compartilhamento de informações e saberes que seria essencial na resolução do processo que levou ao adoecimento. Isso porque os diferentes olhares das profissões permitem evidências até então não percebidas.

A dificuldade de comunicação e formação que valoriza o núcleo sem preparar o profissional para o trabalho interdisciplinar também foram ressaltadas por Lazarino e colaboradores (2019) em seu estudo sobre apoio matricial realizado em Minas Gerais.

Torna-se notório o quanto é importante o conhecimento sobre o processo de trabalho das equipes e as maiores demandas e agravos que abrangem o processo de saúde-doença das pessoas que fazem parte dos territórios sob responsabilidade sanitária da ESF, para o desenvolvimento do matriciamento.

Logo, nota-se o apoio da equipe do NASF às equipes de SF, o suporte da gestão e a possibilidade de um espaço de escuta das queixas e das sugestões das equipes são fundamentais para estimular a integração, sedimentar a implantação do apoio matricial e avaliar continuamente o processo para o aperfeiçoamento e o desenvolvimento de novas estratégias (BRASIL, 2010).

Tais entraves podem ser consequências da falta de formação dos profissionais do NASF para desempenhar o matriciamento. Desta forma, cria-se perspectiva para a qualificação desses profissionais, no viés de intensificar a 
remodelação das práticas em saúde e a consolidação da atenção básica, através apoio matricial no município. Acredita-se assim, atuação de forma interdisciplinar e intersetorial, com o escopo de cessar a desintegração do cuidado ainda presente, apesar das várias discussões sobre a Política de Humanização.

\section{CONSIDERAÇÕES FINAIS}

$O$ relato de experiência permitiu averiguar que só é possível garantir a integralidade do cuidado com uma boa articulação entre equipe de referência e matriciadora. As dificuldades encontradas pelas equipes se referem às divergências na agenda, falta de formação do NASF para apoio matricial, dificuldades relacionadas à padronização de condutas, ao referenciamento e à comunicação. Isso ratifica os resultados de outras pesquisas, que reconheceram graves entraves na troca de informações e referenciamento na APS.

Dessa forma, o trabalho entre NASF e ESF apresentou-se fracionado e com pouca vinculação, indispensável no processo de trabalho. No cenário estudado, o diálogo entre essas duas equipes e a articulação de ações era pouco frequente, com predomínio da atuação do NASF de forma desarticulada, ausência de contra referência, pouco vínculo, atividades pontuais, preferência por consultas especializadas e falta de responsabilização pelo cuidado. Tais fatores comprometeram a resolução de casos e consequentemente o cuidado integral.

O relato traz como contribuição a análise do processo de trabalho entre equipe de saúde da família e NASF, com uma visão que traz subsídios para a reflexão; tendo a intenção de cooperar para o aperfeiçoamento, progresso das ações na APS e a concretização das políticas públicas. Vale ressaltar que o processo de gestão não é responsabilidade apenas de profissionais da saúde ou gestores, mas a participação social é de suma importância para se alcançar um sistema de saúde que garanta o cuidado de forma equânime, universal e integral. 


\section{REFERÊNCIAS}

ALELUIA, Italo Ricardo Santos; MEDINA, Maria Guadalupe; ALMEIDA, Patty Fidelis de; VILASBÔAS, Ana Luiza Queiroz. Coordenação do cuidado na atenção primária à saúde: estudo avaliativo em município sede de macrorregião do nordeste brasileiro. Ciência \& Saúde Coletiva, [s.I.], v. 22, n. 6, p. 1845-1856, jun. 2017. FapUNIFESP (SciELO).

ALMEIDA, Patty Fidelis de; MEDINA, Maria Guadalupe; FAUSTO, Márcia Cristina RodrigueS; GIOVANELLA; BOUSQUAT, Aylene; MENDONÇA, Maria Helena Magalhães de. Coordenação do cuidado e Atenção Primária à Saúde no Sistema Único de Saúde. Saúde debate | Rio de Janeiro, v. 42, número especial 1, p. 244-260, setembro 2018.

BRASIL. Ministério da Saúde. Portaria no 2.436, de 21 de setembro de 2017. Aprova a Política Nacional de Atenção Básica, estabelecendo a revisão de diretrizes para a organização da Atenção Básica, no âmbito do Sistema Único de Saúde (SUS). Brasília, DF: Ministério da Saúde, 2017.

Ministério da Saúde. Secretaria de Atenção à Saúde. Departamento de Atenção Básica. Guia prático de matriciamento em saúde mental / Dulce Helena Chiaverini (Organizadora) ... [et al.]. Brasília, DF 2010. 152 p. : il. - (Série A. Normas e Manuais Técnicos) (Caderno de Atenção Básica, n. 27)

Ministério da Saúde. Secretaria de Atenção à Saúde. Política Nacional de Humanização da Atenção e Gestão do SUS. Clínica ampliada e compartilhada / Ministério da Saúde, Secretaria de Atenção à Saúde, Política Nacional de Humanização da Atenção e Gestão do SUS. - Brasília : Ministério da Saúde, 2009. 64 p. : il. color. - (Série B. Textos Básicos de Saúde)

FAGUNDES, Giselle Soares; CAMPOS, Monica Rodrigues; FORTES, Sandra Lúcia Correia Lima. Matriciamento em Saúde Mental: análise do cuidado às pessoas em sofrimento psíquico na Atenção Básica. Ciência \& Saúde Coletiva, 26(6):2311-2322, 2021.

LAZARINO, Márcia da Silva Anunciação; SILVA, Thais Lacerda e; DIAS, Elizabeth Costa. Apoio matricial como estratégia para o fortalecimento da saúde do trabalhador na atenção básica. Revista Brasileira de Saúde Ocupacional, Belo Horizonte, v. 44, n. 23, p. 1-8, ago. 2019. FapUNIFESP (SciELO).

MEDEIROS, Roberto Henrique Amorim de. Uma noção de matriciamento que merece ser resgatada para o encontro colaborativo entre equipes de saúde e serviços no SUS. Physis: Revista de Saúde Coletiva, [s.I.], v. 25 , n. 4, p. 1165-1184, dez. 2015. FapUNIFESP (SciELO).

OLIVEIRA, Kátia Santos; BADUY, Rossana Staevie; MELCHIOR, Regina. O encontro entre o Núcleo de Apoio à Saúde da Família e as equipes de Saúde da Família: a produção de um coletivo cuidador. Physis: Revista de Saúde Coletiva, Rio de Janeiro, v. 29(4), e290403, 2019

SANTOS, Rosimeire Aparecida Bezerra de Gois dos; UCHÔA-FIGUEIREDO, Lúcia da Rocha; LIMA, Laura Câmara. Apoio matricial e ações na atenção primária: experiência de profissionais de esf e nasf. : experiência de profissionais de ESF e Nasf. Saúde em Debate, [s.I.], v. 41, n. 114, p. 694-706, set. 2017.

SILVA, Joyce Lane Braz Virgolino da; OLIVEIRA, Anderson Belmont Correia de; OLIVEIRA, Ana Giovana Medeiros de; OLIVEIRA, KalígiaDeininger de; OLIVEIRA, Fabíola Moreira Casimiro de; ALVES, Monica Rocha Rodrigues. A prática da integralidade na gestão do cuidado: relato de experiência.Revista de Enfermagem: UFPE ONLINE, Recife, v. 2, n. 11, p. 792-797, fev. 2017. 